\title{
On integral inequalities related to the weighted and the extended Chebyshev functionals involving different fractional operators
}

\author{
Barış Çelik1* (1), Mustafa Ç. Gürbüz², M. Emin Özdemir ${ }^{2}$ and Erhan Set ${ }^{1}$
}

"Correspondence:

bariscelik15@hotmail.com

1 Department of Mathematics,

Faculty of Science and Arts, Ordu

University, Ordu, Turkey

Full list of author information is

available at the end of the article

\section{Springer}

\begin{abstract}
The role of fractional integral operators can be found as one of the best ways to generalize classical inequalities. In this paper, we use different fractional integral operators to produce some inequalities for the weighted and the extended Chebyshev functionals. The results are more general than the available classical results in the literature.
\end{abstract}

MSC: 26A33; 26D10; 26D15

Keywords: Chebyshev's functional; Fractional integral operators

\section{Introduction and preliminaries}

Fractional calculus, which is calculus of integrals and derivatives of any arbitrary real or complex order, has gained remarkable popularity and importance during the last four decades or so, due mainly to its demonstrated applications in diverse and widespread fields ranging from natural sciences to social sciences (see, e.g., [1, 3, 17, 19, 20, 23, 24] and the references therein). Beginning with the classical Riemann-Liouville fractional integral and derivative operators, a large number of fractional integral and derivative operators and their generalizations have been presented. Also, many authors have established a variety of inequalities for those fractional integral and derivative operators, some of which have turned out to be useful in analyzing solutions of certain fractional integral and differential equations.

Definition 1.1 Let $[a, b](-\infty<a<b<\infty)$ be a finite interval on the real axis $\mathbb{R}$. The Riemann-Liouville fractional integrals $J_{a+}^{\alpha} f$ and $J_{b-}^{\alpha} f$ of order $\alpha \in \mathbb{C}(\Re(\alpha)>0)$ with $a \geq 0$ and $b>0$ are defined, respectively, by

$$
\left(J_{a+}^{\alpha} f\right)(x):=\frac{1}{\Gamma(\alpha)} \int_{a}^{x}(x-t)^{\alpha-1} f(t) d t \quad(x>a ; \Re(\alpha)>0)
$$

(c) The Author(s) 2020. This article is licensed under a Creative Commons Attribution 4.0 International License, which permits use sharing, adaptation, distribution and reproduction in any medium or format, as long as you give appropriate credit to the original author(s) and the source, provide a link to the Creative Commons licence, and indicate if changes were made. The images or other third party material in this article are included in the article's Creative Commons licence, unless indicated otherwise in a credit line to the material. If material is not included in the article's Creative Commons licence and your intended use is not permitted by statutory regulation or exceeds the permitted use, you will need to obtain permission directly from the copyright holder. To view a copy of this licence, visit http://creativecommons.org/licenses/by/4.0/. 
and

$$
\left(J_{b-}^{\alpha} f\right)(x):=\frac{1}{\Gamma(\alpha)} \int_{x}^{b}(t-x)^{\alpha-1} f(t) d t \quad(x<b ; \Re(\alpha)>0) .
$$

Here, $\Gamma(\alpha)$ is the familiar gamma function (see, e.g., [28, Sect. 1.1]). For more details and properties concerning the fractional integral operators (1.1) and (1.2), we refer the reader, for example, to the works $[6,13,15,18,19,24,25,27,29,30]$ and the references therein.

In [5], the Chebyshev functional for two integrable functions $f$ and $g$ on $[a, b]$ is defined as follows:

$$
T(f, g)=\frac{1}{b-a} \int_{a}^{b} f(x) g(x) d x-\frac{1}{b-a}\left(\int_{a}^{b} f(x) d x\right) \frac{1}{b-a}\left(\int_{a}^{b} g(x) d x\right) .
$$

In $[3,11,12]$ the applications and several inequalities related to (1.3) are found. In [5], the weighted Chebyshev functional is defined by

$$
\begin{aligned}
T(f, g, p)= & \frac{1}{b-a} \int_{a}^{b} p(x) d x \int_{a}^{b} f(x) g(x) p(x) d x \\
& -\int_{a}^{b} f(x) p(x) d x \int_{a}^{b} g(x) p(x) d x
\end{aligned}
$$

where $f$ and $g$ are integrable on $[a, b]$ and $p$ is a positive and integrable function on $[a, b]$. In [14], Elezovic et al. proved that

$$
\begin{aligned}
|T(f, g, p)| \leq & \frac{1}{2}\left(\left.\left.\int_{a}^{b} \int_{a}^{b} p(x) p(y)|x-y|^{\frac{1}{\alpha^{\prime}}+\frac{1}{\beta^{\prime}}}\left|\int_{y}^{x}\right| f^{\prime}(t)\right|^{\alpha} d t\right|^{\frac{\gamma}{\alpha}} d x d y\right)^{\frac{1}{\gamma}} \\
& \times\left(\left.\left.\int_{a}^{b} \int_{a}^{b} p(x) p(y)|x-y|^{\frac{1}{\alpha^{\prime}}+\frac{1}{\beta^{\prime}}}\left|\int_{y}^{x}\right| g^{\prime}(t)\right|^{\beta} d t\right|^{\frac{\gamma^{\prime}}{\beta}} d x d y\right)^{\frac{1}{\gamma^{\prime}}} \\
\leq & \frac{1}{2}\left\|f^{\prime}\right\|_{\alpha}\left\|g^{\prime}\right\|_{\beta}\left(\int_{a}^{b} \int_{a}^{b} p(x) p(y)|x-y|^{\frac{1}{\alpha^{\prime}}+\frac{1}{\beta^{\prime}}} d x d y\right)
\end{aligned}
$$

where $f^{\prime} \in L^{\alpha}([a, b])$ and $g^{\prime} \in L^{\beta}([a, b]), \alpha, \beta, \gamma>1, \frac{1}{\alpha}+\frac{1}{\alpha^{\prime}}=1, \frac{1}{\beta}+\frac{1}{\beta^{\prime}}=1$, and $\frac{1}{\gamma}+\frac{1}{\gamma^{\prime}}=1$.

In [10], Dahmani et al. proved the following fractional integral inequality for Chebyhev functionals:

$$
\begin{aligned}
& 2\left|J^{\delta} p(t) J^{\delta} p f g(t)-J^{\delta} p f(t) J^{\delta} p g(t)\right| \\
& \quad \leq \frac{\left\|f^{\prime}\right\|_{\alpha}\left\|g^{\prime}\right\|_{\beta}}{\Gamma^{2}(\delta)} \int_{0}^{t} \int_{0}^{t}(t-x)^{\delta-1}(t-y)^{\delta-1}|x-y| p(x) p(y) d x d y,
\end{aligned}
$$

where $f^{\prime} \in L^{\alpha}([0, \infty))$ and $g^{\prime} \in L^{\beta}([0, \infty)), \alpha, \beta>1$ and $\frac{1}{\alpha}+\frac{1}{\beta}=1$.

Additionally, taking a positive and integrable function $q$ on $[a, b]$, we consider the extended Chebyshev functional $[7,22]$

$$
\begin{aligned}
\tilde{T}(f, g, p, q)= & \int_{a}^{b} q(x) d x \int_{a}^{b} f(x) g(x) p(x) d x+\int_{a}^{b} p(x) d x \int_{a}^{b} f(x) g(x) q(x) d x \\
& -\int_{a}^{b} f(x) p(x) d x \int_{a}^{b} g(x) q(x) d x-\int_{a}^{b} f(x) q(x) d x \int_{a}^{b} g(x) p(x) d x
\end{aligned}
$$


Many researchers have given valuable attention to functionals (1.4) and (1.5). For more details, we refer the reader to $[3,8,21]$ and the references therein.

Dahmani et al. [9], established some inequalities for the weighted and the extended Chebyshev functionals with certain conditions via Riemann-Liouville fractional integrals, which are recalled in the following two theorems.

Theorem 1.1 Let $f$ and $g$ be two differentiable functions on $[0, \infty)$ and $p$ be a positive and integrable function on $[0, \infty)$. If $f^{\prime} \in L^{\alpha}([0, \infty)), g^{\prime} \in L^{\beta}([0, \infty)), \alpha, \beta, \gamma>1$ with $\frac{1}{\alpha}+\frac{1}{\alpha^{\prime}}=1$, $\frac{1}{\beta}+\frac{1}{\beta^{\prime}}=1$, and $\frac{1}{\gamma}+\frac{1}{\gamma^{\prime}}=1$, then for all $t>0, \delta>0$, we have the inequality

$$
\begin{aligned}
2\left|J^{\delta} p(t) J^{\delta} p f g(t)-J^{\delta} p g(t) J^{\delta} p f(t)\right| & \\
\leq & \left(\frac{\left\|f^{\prime}\right\|_{\alpha}^{\gamma}}{\Gamma(\delta)} \int_{0}^{t} \int_{0}^{t}(t-x)^{\delta-1}(t-y)^{\delta-1} p(x) p(y)|x-y|^{\frac{1}{\alpha^{\prime}}+\frac{1}{\beta^{\prime}}} d x d y\right)^{\frac{1}{\gamma}} \\
& \times\left(\frac{\left\|g^{\prime}\right\|_{\beta}^{\gamma^{\prime}}}{\Gamma(\alpha)} \int_{0}^{t} \int_{0}^{t}(t-x)^{\delta-1}(t-y)^{\delta-1} p(x) p(y)|x-y|^{\frac{1}{\alpha^{\prime}}+\frac{1}{\beta^{\prime}}} d x d y\right)^{\frac{1}{\gamma^{\prime}}} \\
\leq & \frac{\left\|f^{\prime}\right\|_{\alpha}\left\|g^{\prime}\right\|_{\beta}}{\Gamma(\delta)^{2}}\left(\int_{0}^{t} \int_{0}^{t}(t-x)^{\delta-1}(t-y)^{\delta-1}|x-y|^{\frac{1}{\alpha^{\prime}}+\frac{1}{\beta^{\prime}}} p(x) p(y) d x d y\right) .
\end{aligned}
$$

Theorem 1.2 Let $f$ and $g$ be two differentiable functions on $[0, \infty)$ and $p, q$ be two positive and integrable functions on $[0, \infty)$. If $f^{\prime} \in L^{\alpha}([0, \infty)), g^{\prime} \in L^{\beta}([0, \infty)), \alpha, \beta, \gamma>1$ with $\frac{1}{\alpha}+$ $\frac{1}{\alpha^{\prime}}=1, \frac{1}{\beta}+\frac{1}{\beta^{\prime}}=1$, and $\frac{1}{\gamma}+\frac{1}{\gamma^{\prime}}=1$, then for all $t>0, \delta>0$, we have

$$
\begin{aligned}
& \left|J^{\delta} q(t) J^{\delta} p f g(t)+J^{\delta} p(t) J^{\delta} q f g(t)-J^{\delta} p f(t) J^{\delta} q g(t)-J^{\delta} q f(t) J^{\delta} p g(t)\right| \\
& \quad \leq \frac{\left\|f^{\prime}\right\|_{\alpha}\left\|g^{\prime}\right\|_{\beta}}{\Gamma(\delta)^{2}}\left(\int_{0}^{t} \int_{0}^{t}(t-x)^{\delta-1}(t-y)^{\delta-1}|x-y|^{\frac{1}{\alpha^{\prime}}+\frac{1}{\beta^{\prime}}} p(x) q(y) d x d y\right) .
\end{aligned}
$$

\section{Main results}

In this section we present some inequalities for the weighted and the extended Chebyshev functionals involving the fractional integral operators, respectively, Katugampola fractional integral operator, mixed conformable fractional integral operator, and Hadamard fractional integral operator.

Definition 2.1 ([16]) Let $[a, b] \subset \mathbb{R}$ be a finite interval. Then the left- and right-hand side Katugampola fractional integrals of order $(\alpha>0)$ of $f \in X_{c}^{p}(a, b)$ are defined as follows:

$$
{ }^{\rho} \mathcal{I}_{a+}^{\alpha} f(x)=\frac{\rho^{1-\alpha}}{\Gamma(\alpha)} \int_{a}^{x} \frac{t^{\rho-1}}{\left(x^{\rho}-t^{\rho}\right)^{1-\alpha}} f(t) d t
$$

and

$$
{ }^{\rho} \mathcal{I}_{b-}^{\alpha} f(x)=\frac{\rho^{1-\alpha}}{\Gamma(\alpha)} \int_{x}^{b} \frac{t^{\rho-1}}{\left(t^{\rho}-x^{\rho}\right)^{1-\alpha}} f(t) d t
$$

with $a<x<b$ and $\rho>0$, if the integral exists.

Theorem 2.1 Let $f$ and $g$ be two differentiable functions on $[0, \infty)$ and $p$ be a positive and integrable function on $[0, \infty)$. If $f^{\prime} \in X_{c}^{p}\left(a^{\rho}, b^{\rho}\right), g^{\prime} \in X_{c}^{p}\left(a^{\rho}, b^{\rho}\right), r, s, \gamma>1$ with $\frac{1}{r}+\frac{1}{r^{\prime}}=1$, 
$\frac{1}{s}+\frac{1}{s^{\prime}}=1$, and $\frac{1}{\gamma}+\frac{1}{\gamma^{\prime}}=1$, then for all $t>0, \alpha, \rho>0$, we have

$$
\begin{aligned}
\left.2\right|^{\rho} \mathcal{T}^{\alpha} p(t)^{\rho} \mathcal{I}^{\alpha} p f g(t)-{ }^{\rho} \mathcal{I}^{\alpha} p g(t)^{\rho} \mathcal{I}^{\alpha} p f(t) \mid \\
\leq\left[\frac{\rho^{1-\alpha}\left\|f^{\prime}\right\|_{r}^{\gamma}}{\Gamma(\alpha)} \int_{0}^{t} \int_{0}^{t} \frac{x^{\rho-1}}{\left(t^{\rho}-x^{\rho}\right)^{1-\alpha}} \frac{y^{\rho-1}}{\left(t^{\rho}-y^{\rho}\right)^{1-\alpha}}|x-y|^{\frac{1}{r^{\prime}}+\frac{1}{s^{\prime}}} p(x) p(y) d x d y\right]^{\frac{1}{\gamma}} \\
\quad \times\left[\frac{\rho^{1-\alpha}\left\|g^{\prime}\right\|_{s}^{\gamma^{\prime}}}{\Gamma(\alpha)} \int_{0}^{t} \int_{0}^{t} \frac{x^{\rho-1}}{\left(t^{\rho}-x^{\rho}\right)^{1-\alpha}} \frac{y^{\rho-1}}{\left(t^{\rho}-y^{\rho}\right)^{1-\alpha}}|x-y|^{\frac{1}{r^{\prime}}+\frac{1}{s^{\prime}}} p(x) p(y) d x d y\right]^{\frac{1}{\gamma^{\prime}}} \\
\leq\left(\frac{\rho^{1-\alpha}}{\Gamma(\alpha)}\right)^{2}\left\|f^{\prime}\right\|_{r}\left\|g^{\prime}\right\|_{s} \int_{0}^{t} \int_{0}^{t} \frac{x^{\rho-1}}{\left(t^{\rho}-x^{\rho}\right)^{1-\alpha}} \frac{y^{\rho-1}}{\left(t^{\rho}-y^{\rho}\right)^{1-\alpha}}|x-y|^{\frac{1}{r^{\prime}}+\frac{1}{s^{\prime}}} p(x) p(y) d x d y .
\end{aligned}
$$

Proof Let us define

$$
H(x, y):=(f(x)-f(y)(g(x)-g(y))) ; \quad x, y \in(0, t), t>0 .
$$

Multiplying (2.2) by $\frac{\rho^{1-\alpha}}{\Gamma(\alpha)} \frac{x^{\rho-1}}{\left(t^{\rho}-x^{\rho}\right)^{1-\alpha}} p(x)$ and integrating the resulting identity with respect to $x$ from 0 to $t$, we can write

$$
\begin{aligned}
& \frac{\rho^{1-\alpha}}{\Gamma(\alpha)} \int_{0}^{t} \frac{x^{\rho-1}}{\left(t^{\rho}-x^{\rho}\right)^{1-\alpha}} p(x) H(x, y) d x \\
& \quad={ }^{\rho} \mathcal{I}^{\alpha} p f g(t)-g(y)^{\rho} \mathcal{I}^{\alpha} p f(t)-f(y)^{\rho} \mathcal{I}^{\alpha} p g(t)+f(y) g(y)^{\rho} \mathcal{I}^{\alpha} p(t) .
\end{aligned}
$$

Again, multiplying (2.3) by $\frac{\rho^{1-\alpha}}{\Gamma(\alpha)} \frac{y^{\rho-1}}{\left(t^{\rho}-y^{\rho}\right)^{1-\alpha}} p(y)$ and integrating the resulting identity with respect to $y$ from 0 to $t$, we can write

$$
\begin{aligned}
& \left(\frac{\rho^{1-\alpha}}{\Gamma(\alpha)}\right)^{2} \int_{0}^{t} \int_{0}^{t} \frac{x^{\rho-1}}{\left(t^{\rho}-x^{\rho}\right)^{1-\alpha}} \frac{y^{\rho-1}}{\left(t^{\rho}-y^{\rho}\right)^{1-\alpha}} p(x) p(y) H(x, y) d x d y \\
& \quad=2\left[{ }^{\rho} \mathcal{I}^{\alpha} p(t)^{\rho} \mathcal{I}^{\alpha} p f g(t)-{ }^{\rho} \mathcal{I}^{\alpha} p g(t)^{\rho} \mathcal{I}^{\alpha} p f(t)\right] .
\end{aligned}
$$

Also, on the other hand, we have

$$
H(x, y):=\int_{y}^{x} \int_{y}^{x} f^{\prime}(u) g^{\prime}(w) d u d w .
$$

By employing Hölder's inequality, we have

$$
|f(x)-f(y)| \leq\left.\left.|x-y|^{\frac{1}{r^{\prime}}}\left|\int_{y}^{x}\right| f^{\prime}(u)\right|^{r} d u\right|^{\frac{1}{r}}
$$

and

$$
|g(x)-g(y)| \leq\left.\left.|x-y|^{\frac{1}{s^{\prime}}}\left|\int_{y}^{x}\right| g^{\prime}(w)\right|^{s} d w\right|^{\frac{1}{s}} .
$$

Then, we can estimate $H$ as follows:

$$
|H(x, y)| \leq\left.\left.\left.\left.|x-y|^{\frac{1}{r^{\prime}}+\frac{1}{s^{\prime}}}\left|\int_{y}^{x}\right| f^{\prime}(u)\right|^{r} d u\right|^{\frac{1}{r}}\left|\int_{y}^{x}\right| g^{\prime}(w)\right|^{s} d u\right|^{\frac{1}{s}} .
$$


Therefore, we can write

$$
\begin{aligned}
&\left.2\right|^{\rho} \mathcal{I}^{\alpha} p(t)^{\rho} \mathcal{I}^{\alpha} p f g(t)-{ }^{\rho} \mathcal{I}^{\alpha} p g(t)^{\rho} \mathcal{I}^{\alpha} p f(t) \mid \\
& \leq\left(\frac{\rho^{1-\alpha}}{\Gamma(\alpha)}\right)^{2} \int_{0}^{t} \int_{0}^{t} \frac{x^{\rho-1}}{\left(t^{\rho}-x^{\rho}\right)^{1-\alpha}} \frac{y^{\rho-1}}{\left(t^{\rho}-y^{\rho}\right)^{1-\alpha}} p(x) p(y) \\
& \times\left.\left.\left.\left.|x-y|^{\frac{1}{r^{\prime}}+\frac{1}{s^{\prime}}}\left|\int_{y}^{x}\right| f^{\prime}(u)\right|^{r} d u\right|^{\frac{1}{r}}\left|\int_{y}^{x}\right| g^{\prime}(w)\right|^{s} d w\right|^{\frac{1}{s}} d x d y .
\end{aligned}
$$

By Hölder's inequality for double integral, we obtain

$$
\begin{aligned}
\left.2\right|^{\rho} \mathcal{I}^{\alpha} p(t)^{\rho} \mathcal{I}^{\alpha} p f g(t)-{ }^{\rho} \mathcal{I}^{\alpha} p g(t)^{\rho} \mathcal{I}^{\alpha} p f(t) \mid \\
\leq\left(\frac{\rho^{1-\alpha}}{\Gamma(\alpha)}\right)^{2}\left[\int_{0}^{t} \int_{0}^{t} \frac{x^{\rho-1}}{\left(t^{\rho}-x^{\rho}\right)^{1-\alpha}} \frac{y^{\rho-1}}{\left(t^{\rho}-y^{\rho}\right)^{1-\alpha}}|x-y|^{\frac{1}{r^{\prime}+\frac{1}{s^{\prime}}}}\right. \\
\left.\quad \times\left.\left.\left|\int_{y}^{x}\right| f^{\prime}(u)\right|^{r} d u\right|^{\frac{\gamma}{r}} p(x) p(y) d x d y\right]^{\frac{1}{\gamma}} \\
\quad \times\left[\int_{0}^{t} \int_{0}^{t} \frac{x^{\rho-1}}{\left(t^{\rho}-x^{\rho}\right)^{1-\alpha}} \frac{y^{\rho-1}}{\left(t^{\rho}-y^{\rho}\right)^{1-\alpha}}|x-y|^{\frac{1}{r^{\prime}}+\frac{1}{s^{\prime}}}\right. \\
\left.\quad \times\left.\left.\left|\int_{y}^{x}\right| g^{\prime}(w)\right|^{s} d w\right|^{\frac{\gamma^{\prime}}{s}} p(x) p(y) d x d y\right]^{\frac{1}{\gamma^{\prime}}} .
\end{aligned}
$$

Using the following properties:

$$
\left.\left|\int_{x}^{y}\right| f^{\prime}(u)\right|^{r} d u\left|\leq\left\|f^{\prime}\right\|_{r}^{r},\right| \int_{x}^{y}\left|g^{\prime}(w)\right|^{s} d w \mid \leq\left\|g^{\prime}\right\|_{s}^{s},
$$

(2.9) can be written as

$$
\begin{aligned}
\left.2\right|^{\rho} \mathcal{I}^{\alpha} p(t)^{\rho} \mathcal{I}^{\alpha} p f g(t)-{ }^{\rho} \mathcal{I}^{\alpha} p g(t)^{\rho} \mathcal{I}^{\alpha} p f(t) \mid \\
\leq\left[\frac{\rho^{1-\alpha}\left\|f^{\prime}\right\|_{r}^{\gamma}}{\Gamma(\alpha)} \int_{0}^{t} \int_{0}^{t} \frac{x^{\rho-1}}{\left(t^{\rho}-x^{\rho}\right)^{1-\alpha}} \frac{y^{\rho-1}}{\left(t^{\rho}-y^{\rho}\right)^{1-\alpha}}|x-y|^{\frac{1}{r^{\prime}}+\frac{1}{s^{\prime}}} p(x) p(y) d x d y\right]^{\frac{1}{\gamma}} \\
\quad \times\left[\frac{\rho^{1-\alpha}\left\|g^{\prime}\right\|_{s}^{\gamma^{\prime}}}{\Gamma(\alpha)} \int_{0}^{t} \int_{0}^{t} \frac{x^{\rho-1}}{\left(t^{\rho}-x^{\rho}\right)^{1-\alpha}} \frac{y^{\rho-1}}{\left(t^{\rho}-y^{\rho}\right)^{1-\alpha}}|x-y|^{\frac{1}{r^{\prime}}+\frac{1}{s^{\prime}}} p(x) p(y) d x d y\right]^{\frac{1}{\gamma^{\prime}}} .
\end{aligned}
$$

Therefore

$$
\begin{aligned}
& \left.2\right|^{\rho} \mathcal{I}^{\alpha} p(t)^{\rho} \mathcal{I}^{\alpha} p f g(t)-{ }^{\rho} \mathcal{I}^{\alpha} p g(t)^{\rho} \mathcal{I}^{\alpha} p f(t) \mid \\
& \quad \leq\left(\frac{\rho^{1-\alpha}}{\Gamma(\alpha)}\right)^{2}\left\|f^{\prime}\right\|_{r}\left\|g^{\prime}\right\|_{s} \int_{0}^{t} \int_{0}^{t} \frac{x^{\rho-1}}{\left(t^{\rho}-x^{\rho}\right)^{1-\alpha}} \frac{y^{\rho-1}}{\left(t^{\rho}-y^{\rho}\right)^{1-\alpha}}|x-y|^{\frac{1}{r^{\prime}}+\frac{1}{s^{\prime}}} p(x) p(y) d x d y .
\end{aligned}
$$

This completes the proof.

Theorem 2.2 Let $f$ and $g$ be two differentiable functions on $[0, \infty)$ and $p$, $q$ be positive and integrable functions on $[0, \infty)$. If $f^{\prime} \in X_{c}^{p}\left(a^{\rho}, b^{\rho}\right), g^{\prime} \in X_{c}^{p}\left(a^{\rho}, b^{\rho}\right), r, s, \gamma>1$ with $\frac{1}{r}+\frac{1}{r^{\prime}}=1$, 
$\frac{1}{s}+\frac{1}{s^{\prime}}=1$, and $\frac{1}{\gamma}+\frac{1}{\gamma^{\prime}}=1$, then for all $t>0, \alpha, \rho>0$, we have

$$
\begin{aligned}
\left|{ }^{\rho} \mathcal{I}^{\alpha} q(t)^{\rho} \mathcal{I}^{\alpha} p f g(t)+{ }^{\rho} \mathcal{I}^{\alpha} p(t)^{\rho} \mathcal{I}^{\alpha} q f g(t)-{ }^{\rho} \mathcal{I}^{\alpha} p f(t)^{\rho} \mathcal{I}^{\alpha} q g(t)-{ }^{\rho} \mathcal{I}^{\alpha} q f(t)^{\rho} \mathcal{I}^{\alpha} p g(t)\right| \\
\leq\left(\frac{\rho^{1-\alpha}}{\Gamma(\alpha)}\right)^{2}\left\|f^{\prime}\right\|_{r}\left\|g^{\prime}\right\|_{s} \\
\quad \times \int_{0}^{t} \int_{0}^{t} \frac{x^{\rho-1}}{\left(t^{\rho}-x^{\rho}\right)^{1-\alpha}} \frac{y^{\rho-1}}{\left(t^{\rho}-y^{\rho}\right)^{1-\alpha}}|x-y|^{\frac{1}{r^{+}+\frac{1}{s^{\prime}}}} p(x) q(y) d x d y .
\end{aligned}
$$

Proof Multiplying (2.3) by $\frac{\rho^{1-\alpha}}{\Gamma(\alpha)} \frac{y^{\rho-1}}{\left(t^{\rho}-y^{\rho}\right)^{1-\alpha}} q(y)$ and integrating the resulting identity with respect to $y$ from 0 to $t$, we can write

$$
\begin{aligned}
& \left.\right|^{\rho} \mathcal{I}^{\alpha} q(t)^{\rho} \mathcal{I}^{\alpha} p f g(t)+{ }^{\rho} \mathcal{I}^{\alpha} p(t)^{\rho} \mathcal{I}^{\alpha} q f g(t)-{ }^{\rho} \mathcal{I}^{\alpha} p f(t)^{\rho} \mathcal{I}^{\alpha} q g(t)-{ }^{\rho} \mathcal{I}^{\alpha} q f(t)^{\rho} \mathcal{I}^{\alpha} p g(t) \mid \\
& \leq\left(\frac{\rho^{1-\alpha}}{\Gamma(\alpha)}\right)^{2} \int_{0}^{t} \int_{0}^{t} \frac{x^{\rho-1}}{\left(t^{\rho}-x^{\rho}\right)^{1-\alpha}} \frac{y^{\rho-1}}{\left(t^{\rho}-y^{\rho}\right)^{1-\alpha}}|x-y|^{\frac{1}{r^{\prime}}+\frac{1}{s^{\prime}}} \\
& \quad \times\left.\left.\left.\left.\left|\int_{y}^{x}\right| f^{\prime}(u)\right|^{r} d u\right|^{\frac{1}{r}}\left|\int_{y}^{x}\right| g^{\prime}(w)\right|^{s} d w\right|^{\frac{1}{s}} p(x) q(y) d x d y .
\end{aligned}
$$

Using the same arguments as in the proof of Theorem 2.1, we obtain the desired result.

Definition 2.2 ([1]) Let $f$ be defined on $[a, b]$ and $\alpha \in \mathbb{C}, \operatorname{Re}(\alpha)>0, \rho>0$. Then:

(i) The mixed left conformable fractional integral of $f$ is defined by

$$
{ }_{a}^{b} \mathfrak{J}^{\alpha, \rho} f(x)=\frac{1}{\Gamma(\alpha)} \int_{a}^{x} f(s)\left(\frac{(b-s)^{\rho}-(b-x)^{\rho}}{\rho}\right)^{\alpha-1}(b-s)^{\rho-1} d s
$$

and

(ii) The mixed right conformable fractional integral of $f$ is defined by

$$
a_{\mathfrak{J}_{b}^{\alpha, \rho}} f(x)=\frac{1}{\Gamma(\alpha)} \int_{x}^{b} f(s)\left(\frac{(s-a)^{\rho}-(x-a)^{\rho}}{\rho}\right)^{\alpha-1}(s-a)^{\rho-1} d s
$$

For recent results related to this operators, we refer the reader to $[1,2,4,26]$.

Theorem 2.3 Let $f$ and $g$ be two differentiable functions on $[0, \infty)$ and $p$ be a positive and integrable function on $[0, \infty)$. If $f^{\prime} \in L_{r}\left([0, \infty), g^{\prime} \in L_{s}\left([0, \infty), r, s, \gamma>1\right.\right.$ with $\frac{1}{r}+\frac{1}{r^{\prime}}=1$, $\frac{1}{s}+\frac{1}{s^{\prime}}=1$, and $\frac{1}{\gamma}+\frac{1}{\gamma^{\prime}}=1$, then for all $t>0, \alpha, \rho>0$, we have

$$
\begin{aligned}
\left.2\right|_{0} ^{b} \mathfrak{J}^{\alpha, \rho} p(t)_{0}^{b} \mathfrak{J}^{\alpha, \rho} p f g(t)-{ }_{0}^{b} \mathfrak{J}^{\alpha, \rho} p g(t)_{0}^{b} \mathfrak{J}^{\alpha, \rho} p f(t) \mid \\
\leq\left[\frac{\left\|f^{\prime}\right\|_{r}^{\gamma}}{\Gamma(\alpha)} \int_{0}^{t} \int_{0}^{t}\left(\frac{(b-x)^{\rho}-(b-t)^{\rho}}{\rho}\right)^{\alpha-1}(b-x)^{\rho-1}\right. \\
\left.\quad \times\left(\frac{(b-y)^{\rho}-(b-t)^{\rho}}{\rho}\right)^{\alpha-1}(b-y)^{\rho-1}|x-y|^{\frac{1}{r^{\prime}}+\frac{1}{s^{\prime}}} p(x) p(y) d x d y\right]^{\frac{1}{\gamma}} \\
\quad \times\left[\frac{\left\|g^{\prime}\right\|_{s}^{\gamma^{\prime}}}{\Gamma(\alpha)} \int_{0}^{t} \int_{0}^{t}\left(\frac{(b-x)^{\rho}-(b-t)^{\rho}}{\rho}\right)^{\alpha-1}(b-x)^{\rho-1}\right.
\end{aligned}
$$




$$
\begin{aligned}
& \left.\times\left.\left(\frac{(b-y)^{\rho}-(b-t)^{\rho}}{\rho}\right)^{\alpha-1}(b-y)^{\rho-1}|x-y|\right|^{\frac{1}{r^{\prime}}+\frac{1}{s^{\prime}}} p(x) p(y) d x d y\right]^{\frac{1}{\gamma^{\prime}}} \\
\leq & \frac{\left\|f^{\prime}\right\|_{r}\left\|g^{\prime}\right\|_{s}}{\Gamma^{2}(\alpha)} \int_{0}^{t} \int_{0}^{t}\left(\frac{(b-x)^{\rho}-(b-t)^{\rho}}{\rho}\right)^{\alpha-1}(b-x)^{\rho-1} \\
& \times\left(\frac{(b-y)^{\rho}-(b-t)^{\rho}}{\rho}\right)^{\alpha-1}(b-y)^{\rho-1}|x-y|^{\frac{1}{r^{\prime}}+\frac{1}{s^{\prime}}} p(x) p(y) d x d y .
\end{aligned}
$$

Proof Multiplying (2.2) by $\frac{1}{\Gamma(\alpha)}\left(\frac{(b-x)^{\rho}-(b-t)^{\rho}}{\rho}\right)^{\alpha-1}(b-x)^{\rho-1} p(x)$ and integrating the resulting identity with respect to $x$ from 0 to $t$, we can write

$$
\begin{aligned}
& \frac{1}{\Gamma(\alpha)} \int_{0}^{t}\left(\frac{(b-x)^{\rho}-(b-t)^{\rho}}{\rho}\right)^{\alpha-1}(b-x)^{\rho-1} p(x) H(x, y) d x \\
& \quad={ }_{0}^{b} \mathfrak{J}^{\alpha, \rho} p f g(t)-g(y)_{0}^{b} \mathfrak{J}^{\alpha, \rho} p f(t)-f(y)_{0}^{b} \mathfrak{J}^{\alpha, \rho} p g(t)+f(y) g(y)_{0}^{b} \mathfrak{J}^{\alpha, \rho} p(t) .
\end{aligned}
$$

Now, multiplying $(2.11)$ by $\frac{1}{\Gamma(\alpha)}\left(\frac{(b-y)^{\rho}-(b-t)^{\rho}}{\rho}\right)^{\alpha-1}(b-y)^{\rho-1} p(y)$ and integrating the resulting identity with respect to $y$ from 0 to $t$, we can write

$$
\begin{aligned}
& \frac{1}{\Gamma^{2}(\alpha)} \int_{0}^{t} \int_{0}^{t}\left(\frac{(b-x)^{\rho}-(b-t)^{\rho}}{\rho}\right)^{\alpha-1}(b-x)^{\rho-1} \\
& \quad \times\left(\frac{(b-y)^{\rho}-(b-t)^{\rho}}{\rho}\right)^{\alpha-1}(b-y)^{\rho-1} p(x) p(y) H(x, y) d x d y \\
& \quad=2\left[{ }_{0}^{b} \mathfrak{J}^{\alpha, \rho} p(t)_{0}^{b} \mathfrak{J}^{\alpha, \rho} p f g(t)-{ }_{0}^{b} \mathfrak{J}^{\alpha, \rho} p g(t)_{0}^{b} \mathfrak{J}^{\alpha, \rho} p f(t)\right] .
\end{aligned}
$$

Using the same arguments as in the proof of Theorem 2.1, we obtain the desired result.

Theorem 2.4 Let $f$ and $g$ be two differentiable functions on $[0, \infty)$ and $p, q$ be positive and integrable functions on $[0, \infty)$. Iff $\in L_{r}\left([0, \infty), g^{\prime} \in L_{s}\left([0, \infty), r, s, \gamma>1\right.\right.$ with $\frac{1}{r}+\frac{1}{r^{\prime}}=1$, $\frac{1}{s}+\frac{1}{s^{\prime}}=1$, and $\frac{1}{\gamma}+\frac{1}{\gamma^{\prime}}=1$, then for all $t>0, \alpha, \rho>0$, we have

$$
\begin{aligned}
||_{0}^{b} \mathfrak{J}^{\alpha, \rho} q(t)_{0}^{b} \mathfrak{J}^{\alpha, \rho} p f g(t)+{ }_{0}^{b} \mathfrak{J}^{\alpha, \rho} p(t)_{0}^{b} \mathfrak{J}^{\alpha, \rho} q f g(t) & \\
& -{ }_{0}^{b} \mathfrak{J}^{\alpha, \rho} p f(t)_{0}^{b} \mathfrak{J}^{\alpha, \rho} q g(t)-{ }_{0}^{b} \mathfrak{J}^{\alpha, \rho} q f(t)_{0}^{b} \mathfrak{J}^{\alpha, \rho} p g(t) \mid \\
\leq & \frac{\left\|f^{\prime}\right\|_{r}\left\|g^{\prime}\right\|_{s}}{\Gamma^{2}(\alpha)} \int_{0}^{t} \int_{0}^{t}\left(\frac{(b-x)^{\rho}-(b-t)^{\rho}}{\rho}\right)^{\alpha-1}(b-x)^{\rho-1} \\
& \times\left(\frac{(b-y)^{\rho}-(b-t)^{\rho}}{\rho}\right)^{\alpha-1}(b-y)^{\rho-1}|x-y|^{\frac{1}{r^{\prime}}+\frac{1}{s^{\prime}}} p(x) q(y) d x d y .
\end{aligned}
$$

Proof Multiplying (2.11) by $\frac{1}{\Gamma(\alpha)}\left(\frac{(b-y)^{\rho}-(b-t)^{\rho}}{\rho}\right)^{\alpha-1}(b-y)^{\rho-1} q(y)$ and integrating the resulting identity with respect to $y$ from 0 to $t$, we can write

$$
\begin{aligned}
\mid{ }_{0}^{b} \mathfrak{J}^{\alpha, \rho} q(t)_{0}^{b} \mathfrak{J}^{\alpha, \rho} p f g(t)+{ }_{0}^{b} \mathfrak{J}^{\alpha, \rho} p(t)_{0}^{b} \mathfrak{J}^{\alpha, \rho} q f g(t) & \\
& -{ }_{0}^{b} \mathfrak{J}^{\alpha, \rho} p f(t)_{0}^{b} \mathfrak{J}^{\alpha, \rho} q g(t)-{ }_{0}^{b} \mathfrak{J}^{\alpha, \rho} q f(t)_{0}^{b} \mathfrak{J}^{\alpha, \rho} p g(t) \mid \\
\leq & \frac{1}{\Gamma^{2}(\alpha)} \int_{0}^{t} \int_{0}^{t}\left(\frac{(b-x)^{\rho}-(b-t)^{\rho}}{\rho}\right)^{\alpha-1}(b-x)^{\rho-1}
\end{aligned}
$$




$$
\begin{aligned}
& \times\left(\frac{(b-y)^{\rho}-(b-t)^{\rho}}{\rho}\right)^{\alpha-1}(b-y)^{\rho-1}|x-y|^{\frac{1}{r^{\prime}}+\frac{1}{s^{\prime}}} \\
& \times\left.\left.\left.\left.\left|\int_{y}^{x}\right| f^{\prime}(u)\right|^{r} d u\right|^{\frac{1}{r}}\left|\int_{y}^{x}\right| g^{\prime}(w)\right|^{s} d w\right|^{\frac{1}{s}} p(x) q(y) d x d y .
\end{aligned}
$$

Using the same arguments as in the proof of Theorem 2.1, we obtain the desired result.

Definition 2.3 The Hadamard fractional integral of order $\alpha \in \mathbb{R}^{+}$of a function $f(t)$, for all $t>1$, is defined as follows [19]:

$$
{ }_{H} J^{\alpha}\{f(t)\}=\frac{1}{\Gamma(\alpha)} \int_{1}^{t}\left(\log \frac{t}{\tau}\right)^{\alpha-1} f(\tau) \frac{d \tau}{\tau} .
$$

Theorem 2.5 Let $f$ and $g$ be two differentiable functions on $[1, \infty)$ and $p$ be a positive and integrable function on $[1, \infty)$. If $f^{\prime} \in L_{r}\left([1, \infty), g^{\prime} \in L_{s}\left([1, \infty), r, s, \gamma>1\right.\right.$ with $\frac{1}{r}+\frac{1}{r^{\prime}}=1$, $\frac{1}{s}+\frac{1}{s^{\prime}}=1$, and $\frac{1}{\gamma}+\frac{1}{\gamma^{\prime}}=1$, then for all $t>1, \alpha>0$, we have

$$
\begin{aligned}
\left.2\right|_{H} J^{\alpha} & \{p(t)\}_{H} J^{\alpha}\{p f g(t)\}-{ }_{H} J^{\alpha}\{p g(t)\}_{H} J^{\alpha}\{p f(t)\} \mid \\
\leq & {\left[\frac{\left\|f^{\prime}\right\|_{r}^{\gamma}}{\Gamma(\alpha)} \int_{1}^{t} \int_{1}^{t}\left(\log \frac{t}{x}\right)^{\alpha-1}\left(\log \frac{t}{y}\right)^{\alpha-1}|x-y|^{\frac{1}{r^{\prime}}+\frac{1}{s^{\prime}}} \frac{p(x) p(y)}{x y} d x d y\right]^{\frac{1}{\gamma}} } \\
& \times\left[\frac{\left\|g^{\prime}\right\|_{s}^{\gamma^{\prime}}}{\Gamma(\alpha)} \int_{1}^{t} \int_{1}^{t}\left(\log \frac{t}{x}\right)^{\alpha-1}\left(\log \frac{t}{y}\right)^{\alpha-1}|x-y|^{\frac{1}{r^{\prime}}+\frac{1}{s^{\prime}}} \frac{p(x) p(y)}{x y} d x d y\right]^{\frac{1}{\gamma^{\prime}}} \\
\leq & \frac{\left\|f^{\prime}\right\|_{r}\left\|_{g^{\prime}}\right\|_{s}}{\Gamma^{2}(\alpha)} \int_{1}^{t} \int_{1}^{t}\left(\log \frac{t}{x}\right)^{\alpha-1}\left(\log \frac{t}{y}\right)^{\alpha-1}|x-y|^{\frac{1}{r^{\prime}}+\frac{1}{s^{\prime}}} \frac{p(x) p(y)}{x y} d x d y .
\end{aligned}
$$

Proof Multiplying (2.2) by $\frac{\left(\log \frac{t}{x}\right)^{\alpha-1}}{x \Gamma(\alpha)} p(x)$ and integrating the resulting identity with respect to $x$ from 1 to $t$, we can write

$$
\begin{aligned}
& \frac{1}{\Gamma(\alpha)} \int_{1}^{t}\left(\log \frac{t}{x}\right)^{\alpha-1} \frac{p(x)}{x} H(x, y) d x \\
& \quad={ }_{H} J^{\alpha}\{p f g(t)\}-g(y)_{H} J^{\alpha}\{p f(t)\}-f(y)_{H} J^{\alpha}\{p g(t)\}+f(y) g(y)_{H} J^{\alpha}\{p(t)\} .
\end{aligned}
$$

Now, multiplying $(2.12)$ by $\frac{\left(\log \frac{t}{y}\right)^{\alpha-1}}{y \Gamma(\alpha)} p(y)$ and integrating the resulting identity with respect to $y$ from 1 to $t$, we can write

$$
\begin{gathered}
\frac{1}{\Gamma^{2}(\alpha)} \int_{1}^{t} \int_{1}^{t}\left(\log \frac{t}{x}\right)^{\alpha-1}\left(\log \frac{t}{y}\right)^{\alpha-1} \frac{p(x) p(y)}{x y} H(x, y) d x d y \\
=2\left[{ }_{H} J^{\alpha}\{p(t)\}_{H} J^{\alpha}\{p f g(t)\}-{ }_{H} J^{\alpha}\{p g(t)\}_{H} J^{\alpha}\{p f(t)\}\right] .
\end{gathered}
$$

Using the same arguments as in the proof of Theorem 2.1, we obtain the desired result.

Theorem 2.6 Let $f$ and $g$ be two differentiable functions on $[1, \infty)$ and $p, q$ be positive and integrable functions on $[1, \infty)$. Iff $f^{\prime} \in L_{r}\left([1, \infty), g^{\prime} \in L_{s}\left([1, \infty), r, s, \gamma>1\right.\right.$ with $\frac{1}{r}+\frac{1}{r^{\prime}}=1$, 
$\frac{1}{s}+\frac{1}{s^{\prime}}=1$, and $\frac{1}{\gamma}+\frac{1}{\gamma^{\prime}}=1$, then for all $t>1, \alpha>0$, we have

$$
\begin{aligned}
& \mid{ }_{H} J^{\alpha}\{q(t)\}_{H} J^{\alpha}\{p f g(t)\}+{ }_{H} J^{\alpha}\{p(t)\}_{H} J^{\alpha}\{q f g(t)\} \\
&-{ }_{H} J^{\alpha}\{p f(t)\}_{H} J^{\alpha}\{q g(t)\}-{ }_{H} J^{\alpha}\{q f(t)\}_{H} J^{\alpha}\{p g(t)\} \mid \\
& \leq \frac{\left\|f^{\prime}\right\|_{r}\left\|g^{\prime}\right\|_{s}}{\Gamma^{2}(\alpha)} \int_{1}^{t} \int_{1}^{t}\left(\log \frac{t}{x}\right)^{\alpha-1}\left(\log \frac{t}{y}\right)^{\alpha-1}|x-y|^{\frac{1}{r^{\prime}}+\frac{1}{s^{\prime}}} \frac{p(x) p(y)}{x y} d x d y .
\end{aligned}
$$

Proof Multiplying (2.12) by $\frac{\left(\log \frac{t}{y}\right)^{\alpha-1}}{y \Gamma(\alpha)} q(y)$ and integrating the resulting identity with respect to $y$ from 1 to $t$, we can write

$$
\begin{aligned}
\mid{ }_{H} J^{\alpha}\{q(t)\}_{H} J^{\alpha}\{p f g(t)\}+{ }_{H} J^{\alpha}\{p(t)\}_{H} J^{\alpha}\{q f g(t)\} \\
-{ }_{H} J^{\alpha}\{p f(t)\}_{H} J^{\alpha}\{q g(t)\}-{ }_{H} J^{\alpha}\{q f(t)\}_{H} J^{\alpha}\{p g(t)\} \mid \\
\leq \frac{1}{\Gamma^{2}(\alpha)} \int_{1}^{t} \int_{1}^{t}\left(\log \frac{t}{x}\right)^{\alpha-1}\left(\log \frac{t}{y}\right)^{\alpha-1}|x-y|^{\frac{1}{r^{\prime}}+\frac{1}{s^{\prime}}} \\
\quad \times\left.\left.\left.\left.\left|\int_{y}^{x}\right| f^{\prime}(u)\right|^{r} d u\right|^{\frac{1}{r}}\left|\int_{y}^{x}\right| g^{\prime}(w)\right|^{s} d w\right|^{\frac{1}{s}} \frac{p(x) q(y)}{x y} d x d y .
\end{aligned}
$$

Using the same arguments as in the proof of Theorem 2.1, we obtain the desired result.

\section{Concluding remarks}

In this paper, we established some integral inequalities related to the weighted and the extended Chebyshev functionals for different fractional integral operators. If we consider $\rho=1$ in Theorem 2.1 and Theorem 2.2, then the obtained results will reduce to the said inequalities obtained by Dahmani et al. [9].

\section{Acknowledgements \\ Not applicable. \\ Funding \\ There is no funding for this work.}

Availability of data and materials

Data sharing is not applicable to this paper as no datasets were generated or analyzed during the current study.

Competing interests

The authors declare that they have no competing interests.

Authors' contributions

All authors jointly worked on the results and they read and approved the final manuscript.

Author details

'Department of Mathematics, Faculty of Science and Arts, Ordu University, Ordu, Turkey. ${ }^{2}$ Department of Mathematics Education, Education Faculty, Bursa Uludağ University, Görükle Campus, Bursa, Turkey.

\section{Publisher's Note}

Springer Nature remains neutral with regard to jurisdictional claims in published maps and institutional affiliations. 


\section{References}

1. Abdeljawad, T:: On conformable fractional calculus. J. Comput. Appl. Math. 279, 57-66 (2015)

2. Abdeljawad, $T$.: Fractional operators with boundary points dependent kernels and integration by parts. Discrete Contin. Dyn. Syst., Ser. S 13(3), 351-375 (2020). https://doi.org/10.3934/dcdss.2020020

3. Belarbi, S., Dahmani, Z:: On some new fractional integral inequalities. J. Inequal. Pure Appl. Math. 10(3), 86 (2009)

4. Çelik, B., Set, E., Akdemir, A.O.: Mixed conformable fractional Grüss-type inequalities. In: 2nd International Conference on Life and Engineering Sciences (ICOLES 2019), Book of Abstracts, Istanbul, Turkey (2019)

5. Chebyshev, P.L.: Sur les expressions approximatives des integrales definies par les autres prises entre les mêmes limites. Proc. Math. Soc. Charkov 2, 93-98 (1882)

6. Chen, F.: On Hermite-Hadamard type inequalities for Riemann-Liouville fractional integrals via two kinds of convexity. Chin. J. Math. 2014, Article ID 173923 (2014)

7. Dahmani, Z: New inequalities in fractional integrals. Int. J. Nonlinear Sci. 9(4), 493-497 (2010)

8. Dahmani, Z:: About some integral inequalities using Riemann-Liouville integrals. Gen. Math. 20(4), 63-69 (2012)

9. Dahmani, Z., Khameli, A., Freha, K.: Some RL-integral inequalities for the weighted and the extended Chebyshev functionals. Konuralp J. Math. 5(1), 43-48 (2017)

10. Dahmani, Z., Mechouar, O., Brahami, S.: Certain inequalities related to the Chebyshev's functional involving Riemann-Liouville operator. Bull. Math. Anal. Appl. 3(4), 38-44 (2011)

11. Dahmani, Z., Tabharit, L., Taf, S.: New inequalities via Riemann-Liouville fractional integration. J. Adv. Res. Sci. Comput. 2(1), 40-45 (2010)

12. Dragomir, S.S.: Some integral inequalities of Grüss type. Indian J. Pure Appl. Math. 31(4), 397-415 (2002)

13. Ekinci, A., Özdemir, M.E.: Some new integral inequalities via Riemann Liouville integral operators. Appl. Comput. Math. 3, 288-295 (2019)

14. Elezovic, N., Marangunic, L.J., Pecaric, J.: Some improvements of Grüss type inequality. J. Math. Inequal. 1, 425-436 (2007)

15. Gorenflo, R., Mainardi, F.: Fractional Calculus: Integral and Differential Equations of Fractional Order, pp. 223-276. Springer, Wien (1997)

16. Katugampola, U.N.: New approach to a generalized fractional integral. Appl. Math. Comput. 218(3), 860-865 (2011)

17. Khalil, R., Al Horani, M., Yousef, A., Sababheh, M.: A new definition of fractional derivative. J. Comput. Appl. Math. 264, 65-70 (2014)

18. Khan, M.A., Khan, T.U.: Parameterized Hermite-Hadamard type inequalities for fractional integrals. Turk. J. Inequal. 1(1), 26-37 (2017)

19. Kilbas, A.A., Srivastava, H.M., Trujillo, J.J.: Theory and Applications of Fractional Differential Equations. North-Holland Mathematics Studies, vol. 204. Elsevier, Amsterdam (2006)

20. Kiryakova, V:: Generalized Fractional Calculus and Applications. Pitman Research Notes in Mathematics Series, vol. 301. Longman, Harlow (1994)

21. McD Mercer, A.: An improvement of the Grüss inequality. JPAM. J. Inequal. Pure Appl. Math. 10(4), 93 (2005)

22. Mitrinovic, D.S.: Analytic Inequalities. Springer, Berlin (1970)

23. Ntouyas, K.S., Agarwal, P., Tariboon, J.: On Polya-Szego and Chebyshev types inequalities involving the Riemann-Liouville fractional integral operators. J. Math. Inequal. 10(2), 491-504 (2016)

24. Podlubni, I.: Fractional Differential Equations. Academic Press, San Diego (1999)

25. Sarıaya, M.Z., Set, E., Yaldız, H., Başak, N.: Hermite-Hadamard's inequalities for fractional integrals and related fractional inequalities. Math. Comput. Model. 57, 2403-2407 (2013)

26. Set, E.: New inequalities of Chebyshev type for mixed conformable fractional integral operators. In: 2nd International Conference on Life and Engineering Sciences (ICOLES 2019), Book of Abstracts, Istanbul, Turkey (2019)

27. Set, E., Çelik, B.: Certain Hermite-Hadamard type inequalities associated with conformable fractional integral operators. Creative Math. Inform. 26(3), 321-330 (2017)

28. Srivastava, H.M., Choi, J.: Zeta and q-Zeta Functions and Associated Series and Integrals. Elsevier, Amsterdam (2012)

29. Wang, J., Li, X., Zhou, Y.: Hermite-Hadamard inequalities involving Riemann-Liouville fractional integrals via s-convex functions and applications to special means. Filomat 30(5), 1143-1150 (2016)

30. Yaldız, H., Akdemir, A.O.: Katugampola fractional integrals within the class of convex functions. Turk. J. Sci. 3, 40-50 (2018)

\section{Submit your manuscript to a SpringerOpen ${ }^{\circ}$ journal and benefit from:}

- Convenient online submission

Rigorous peer review

- Open access: articles freely available online

- High visibility within the field

- Retaining the copyright to your article

Submit your next manuscript at $\boldsymbol{~ s p r i n g e r o p e n . c o m ~}$ 